\title{
«Programme Gestion et impacts du changement climatique : restitution des projets de 2003 »
}

\section{Compte rendu de colloque (Paris, 27 mai 2008)}

\author{
Marie-Ange Baudoin \\ Doctorante en gestion de l'environnement, Centre d'études du développement durable (CEDD), Université libre de Bruxelles, \\ Campus du Solbosch, CP130/02, avenue Franklin D. Roosevelt 50, 1050 Bruxelles, Belgique
}

Le programme Gestion et impacts du changement climatique $(\mathrm{GICC})^{1}$ a organisé sur deux journées un colloque de restitution des projets retenus en 2003 et achevés en 2007 et un séminaire à mi-parcours de présentation des premiers résultats des projets de 2005. Le texte qui suit ne rend compte que des projets achevés en 2007 (première journée) - les projets de 2005 (deuxième journée) n'étant pas finalisés ${ }^{2}$. Il donne également à la fin quelques remarques ou réflexions d'ordre général à propos du programme.

Le colloque du premier jour, qui avait comme titre «Les sciences économiques et sociales en appui aux politiques publiques : négociations internationales, atténuation, adaptation au changement climatique », a été ponctué par la présentation de plusieurs projets, suivie de séances de discussions. L'originalité de ce colloque résidait dans son ambition de réunir des décideurs politiques aux côtés d'experts scientifiques issus des branches «pures » et «molles » de la science. L'approche se voulait interdisciplinaire, en raison des orateurs présents et des projets présentés. La séance d'ouverture a été présidée par Éric Vindimian, président du Comité d'orientation du programme GICC. Il a rappelé les objectifs du programme qui sont de développer des recherches sur les impacts du changement climatique afin d'inciter les décideurs poli-

\footnotetext{
Auteur correspondant : marie-ange.baudoin@ulb.ac.be

${ }^{1}$ Ce programme, lancé en 1999, est piloté par le ministère de l'Écologie, de l'Énergie, du Développement durable et de l'Aménagement du territoire (MEEDDAT) et il est soutenu par l'Agence de l'environnement et de la maîtrise de l'énergie (ADEME) et l'Observatoire national des effets du réchauffement climatique (ONERC). Ses objectifs sont de "développer les connaissances en appui aux politiques publiques en considérant les changements climatiques sous l'angle aussi bien de leurs impacts que des mesures d'atténuation des émissions de gaz à effet de serre et d'adaptation au changement climatique ».

${ }^{2}$ La restitution de ces projets est prévue en novembre 2009.
}

tiques à prendre en compte le climat. Ces objectifs sont fondamentaux étant donnée l'inertie dont font preuve certains décideurs pour intégrer cette problématique dans leurs décisions politiques. É. Vindimian a ensuite conclu son introduction en insistant sur l'importance grandissante des enjeux climatiques dans le monde politique.

Dans la première session, « Négociations internationales », les deux premiers projets présentés reposaient sur des modélisations. L'un visait à étudier les stratégies des marchés énergétiques par le biais de la théorie des jeux (Alain Haurie, Université de Genève), mais ce modèle reste perfectible car il repose sur une hypothèse non vérifiée (la sensibilité exacte du climat aux rejets de gaz à effet de serre, inconnue actuellement, devrait être précisée d'ici 2025) et il ne prend pas en compte des paramètres sociaux, tels que le rôle des décideurs politiques ou des acteurs privés sur le marché des prix pétroliers. Le deuxième projet proposait une approche économiste pour analyser les comportements énergétiques de deux pays en développement, l'Inde et le Brésil (Sandrine Mathy, Centre international de recherche sur l'environnement et le développement [CIRED]). Il a été souligné entre autres la nécessité de réformes dans le système de réduction des émissions de $\mathrm{CO}_{2}$ de Kyoto. Une étude comportementale des décideurs politiques aurait dû être incluse dans ce projet. Était également présentée une recherche prospective juridique de l'efficacité des mécanismes d'observance du Protocole de Kyoto (Sandrine Maljean-Dubois, CNRS / Centre d'études et de recherches internationales et communautaires). Cette étude a mis en évidence que le protocole, bien qu'il soit le système actuel le plus poussé en matière de règlementation de l'environnement, connaît de nombreuses faiblesses de fonctionnement. Une table ronde a terminé la session : Béatrice Adoléhoumé (MEEDDAT) a souligné les besoins en expertises et en recherches, Marc Gillet (ONERC), l'importance grandissante des recherches en économie et le 
manque de coordination entre chercheurs et décideurs, ce qui, a rappelé É. Vindimian, est l'un des objectifs du GICC. Richard Baron (Agence internationale de l'énergie [AIE]) a insisté sur la nécessité d'impliquer diverses disciplines dans l'étude du changement climatique, afin de garantir l'effectivité des mesures environnementales du post-Kyoto. Enfin, Pierre Radanne (expert indépendant) a clôturé ce tour de table en rappelant de façon très claire que les besoins de recherches en sciences sociales ne touchent pas uniquement l'économie, mais aussi l'histoire, la psychologie ou les sciences politiques et que ce type d'approche devrait favoriser l'adhésion des pays en développement à la lutte mondiale contre le changement climatique.

La session de l'après-midi débutait par un résumé du bilan global du GICC : Claude Millier, président du Conseil scientifique du programme GICC, a souligné le caractère nouveau de certaines thématiques, comme la santé, le transport et la biodiversité. Il a évoqué la nécessité de développer une approche plus régionale de la prise en main du changement climatique et de l'adaptation. Le projet "Gestion territoriale du changement climatique » (François Bertrand, Université de Tours) a abordé cet aspect $^{3}$ et a mis en avant le manque d'opérationnalisation de la gestion durable à l'échelle territoriale et l'absence de participation de la population. Le fait que l'adaptation reste trop souvent le domaine des techniciens et non du monde politique a été souligné. La question des transports a été présentée comme étant « l'impensé » des politiques français dans la lutte contre le changement climatique. C. Millier est alors intervenu pour insister sur l'utilité d'établir des campagnes de sensibilisation et d'éducation au changement climatique, notamment à l'échelle du territoire. Cette remarque a rappelé le besoin de recourir aux sciences de l'éducation, malheureusement très peu mentionnées durant le colloque. Enfin, l'exposé a souligné les lacunes au niveau des collaborations entre échelles de décision (nationale et territoriale).

La journée s'est terminée par l'intervention de Guillaume Sainteny (MEEDDAT), qui a souligné l'actuel retard des politiques publiques dans la prise en compte de l'adaptation. Afin de placer davantage les résultats de la recherche à la portée des décideurs, il a préconisé de soutenir la vulgarisation, tout comme l'étude des coûts de l'adaptation et de l'inaction.

En conclusion, plusieurs remarques s'imposent. L'un des points positifs réside dans la présentation de projets faisant appel à de nombreuses disciplines. Cependant, l'interdisciplinarité n'était pas suffisamment présente à l'intérieur des projets et la nécessité de renforcer la place des sciences humaines et sociales dans la recherche sur le climat a été répétée plusieurs fois. Certes, cette nécessité est réelle en France, mais une approche plus internationale

\footnotetext{
3 Pour plus d'informations sur ce projet, $c f$. http://citeres. univ-tours.fr//p_vst/contrats/rapports_finaux.doc
}

du sujet aurait pu tempérer cette critique, puisqu'il existe en Europe des instituts qui étudient l'adaptation sous l'angle des sciences sociales, ce qui n'a pas été évoqué durant ce colloque. Certaines synergies avec ces instituts devraient être envisagées, afin, notamment, de profiter de méthodologies de recherche déjà élaborées. Par exemple, le Tyndall Centre ${ }^{4}$ propose une approche pluridisciplinaire du climat, comprenant des études sur le rôle de la religion ou de certaines valeurs culturelles en tant que frein à l'adaptation.

Ce colloque a permis de souligner les différents domaines qui devront faire l'objet de davantage de recherches au sein du GICC (nouvelles technologies, approches psychologiques et historiques, adaptation, etc.). Les questions de la conscientisation des populations et des déterminants qui favorisent les changements de comportement devront aussi être davantage étudiées. Sciences politiques, sociales, psychologiques ou de l'éducation devront être mobilisées afin, d'une part, de vulgariser les résultats des recherches auprès des décideurs et des populations et, d'autre part, d'évaluer les solutions qui seront approuvées le plus facilement. Cette approche intégrale de l'étude du changement climatique et de ses conséquences, qui, certes, nécessite des recherches de longue haleine, n'a pas été mentionnée lors des diverses interventions. En termes de vulgarisation des résultats, certaines analyses ont été présentées sous forme de modèles et de scénarios, sans toujours indiquer lequel pouvait être considéré comme le meilleur.

Enfin, aucun projet ne s'est conclu sur une présentation claire et explicite $\mathrm{d}^{\prime}$ actions concrètes, à la portée des décideurs (par ailleurs peu présents). Or, il s'agit de l'objectif même du programme GICC : appuyer les politiques publiques dans leurs stratégies d'action contre l'augmentation des gaz à effet de serre et dans leurs choix de mesures d'adaptation. Les participants ont d'ailleurs plusieurs fois exprimé le besoin d'une collaboration plus étroite avec les décideurs. On pourrait pour cela envisager des ateliers régionaux qui réuniraient les scientifiques et les responsables politiques locaux et dans lesquels seraient présentés les résultats des recherches sous forme d'actions concrètes ${ }^{5}$.

Les présentations des divers intervenants sont disponibles sur le site du programme GICC $: \mathrm{http}: / / \mathrm{www}$. gip-ecofor.org/gicc [événements ; événements passés ; Colloque de restitution de l'APR 2003 \& Séminaire à miparcours APR 2005 - présentation des $1^{\text {ers }}$ résultats].

\footnotetext{
${ }^{4}$ Centre de recherche sur le changement climatique et ses impacts. Situé en Grande-Bretagne, le centre est dirigé par Neil Adger et regorge d'une abondante publication dans le domaine de l'adaptation au changement climatique (http://www.tyndall. ac.uk/).

${ }^{5}$ Depuis ce colloque, ces ateliers régionaux sont devenus la règle dans les projets pour lesquels cette confrontation est opportune.
} 